\title{
Neutralino properties in the light of a further indication of an annual modulation effect in WIMP direct search
}

\author{
A. Bottino, ${ }^{1, *}$ F. Donato, ${ }^{1, \dagger}$ N. Fornengo, ${ }^{1, \ddagger}$ and S. Scopel ${ }^{2, \S}$ \\ ${ }^{1}$ Dipartimento di Fisica Teorica, Università di Torino \\ and INFN, Sezione di Torino, Via P. Giuria 1, 10125 Torino, Italy \\ ${ }^{2}$ Instituto de Física Nuclear y Altas Energías, Facultad de Ciencias, Universidad de Zaragoza, Plaza de San Francisco s/n, \\ 50009 Zaragoza, Spain
}

(Received 1 September 1998; published 30 March 1999)

\begin{abstract}
We demonstrate that the further indication of a possible annual modulation effect, singled out by the DAMA/NaI experiment for WIMP direct detection is widely compatible with an interpretation in terms of a relic neutralino as the major component of dark matter in the Universe. We discuss the supersymmetric features of this neutralino in the minimal supersymmetric extension of the standard model and their implications for searches at accelerators. [S0556-2821(99)03105-7]
\end{abstract}

PACS number(s): 12.60.Jv, 11.30.Pb, 14.80.Ly, 95.35.+d

\section{INTRODUCTION}

In the seminal papers of Refs. [1,2] it was pointed out that the Earth's motion around the Sun can produce a sizable annual modulation of the signal in experiments of direct search for heavy relic particles. Actually, the analysis of a new set of data, recently collected by the DAMA/NaI Collaboration (in the period denoted by the Collaboration as running period No. 2) [3] supports the possible presence of an annual modulation effect in the counting rate for weakly interacting massive particles (WIMPs): the hypothesis of the presence of modulation against the hypothesis of the absence of modulation is statistically favored at $98.5 \%$ C.L. The remarkable features of this measurement, obtained with an exposure of $14962 \mathrm{~kg} \times$ day, are the following.

(i) An analysis of the experimental data, based on a maximum likelihood method, pins down, at a $2 \sigma$ C.L., a well delimited region in the plane $\xi \sigma_{\text {scalar }}^{\text {(nucleon }}-m_{\chi}$, where $m_{\chi}$ is the WIMP mass, $\sigma_{\text {scalar }}^{\text {(nucleon) }}$ is the WIMP-nucleon scalar elastic cross section, and $\xi=\rho_{\chi} / \rho_{l}$ is the fractional amount of local WIMP density $\rho_{\chi}$ with respect to the total local dark matter density $\rho_{l}$. This $\xi \sigma_{\text {scalar }}^{\text {(nucleon }}-m_{\chi}$ modulation region is shown in Fig. 1, which is reproduced here from Fig. 6 of Ref. [3] (the values of $\xi \sigma_{\text {scalar }}^{\text {(nucleon) }}$ plotted in Fig. 1 are normalized to the value $\left.\rho_{l}=0.3 \mathrm{GeV} \mathrm{cm}^{-3}\right)$. The ensuing 1 $-\sigma$ ranges for the two quantities are $m_{\chi}=59_{-14}^{+22} \mathrm{GeV}$ and $\xi \sigma_{\text {scalar }}^{\text {(nucleon) }}=7.0_{-1.7}^{+0.4} \times 10^{-9} \mathrm{nb}[3]$.

(ii) The new data confirm a previous indication of an annual modulation (at the $90 \%$ C.L.) found by the same Collaboration, by using a smaller sample of data, collected in the running period No. 1, with an exposure of $4549 \mathrm{~kg} \times$ day [4]. Most remarkably the 2- $\sigma$ C.L. region from data of Ref. [3] is entirely contained inside the $90 \%$ C.L. region derived from data of Ref. [4], also shown in Fig. 1 (the open solid

\footnotetext{
*Email address: bottino@to.infn.it

${ }^{\dagger}$ Email address: donato@to.infn.it

*Email address: fornengo@to.infn.it

${ }^{\S}$ Email address: scopel@ posta.unizar.es
}

curve denotes the 90\% C.L. upper bound derived in Ref. [5], by using pulse shape analysis).

(iii) Because of the property at point (ii), when the data of the two running periods (with a total exposure of 19511 $\mathrm{kg} \times$ day) are combined together, one obtains a more delimited 2- $\sigma$ C.L. region in the plane $\xi \sigma_{\text {scalar }}^{\text {(nucleon) }}-m_{\chi}$, which is fully embedded in the previous regions. Consequently, the determination of $m_{\chi}$ and $\xi \sigma_{\text {scalar }}^{\text {(nucleon }}$ remains very stable: $m_{\chi}$ $=59_{-14}^{+17} \mathrm{GeV}$ and $\xi \sigma_{\text {scalar }}^{\text {(nucleon) }}=7.0_{-1.2}^{+0.4} \times 10^{-9}$ nb (if $\rho_{l}$ is normalized to the value $\rho_{l}=0.3 \mathrm{GeV} \mathrm{cm}^{-3}$ ). By combining the two sets of data, the hypothesis of presence of modu-

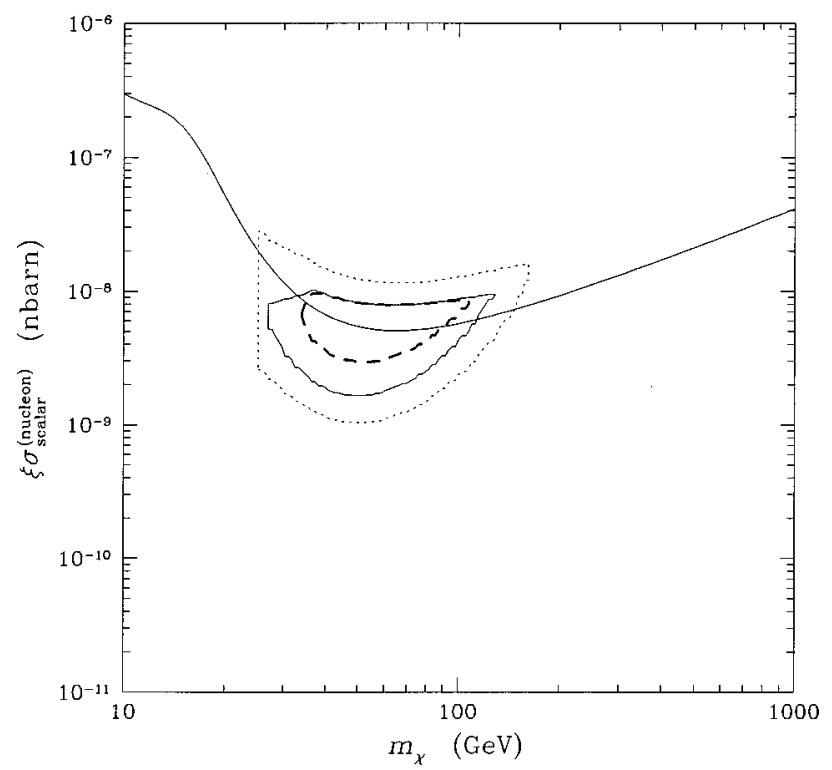

FIG. 1. Annual modulation regions singled out by the DAMA/ $\mathrm{NaI}$ experiments in the plane $m_{\chi}-\xi \sigma_{\text {scalar }}^{\text {(nucleon })}$. The dotted contour line denotes the $90 \%$ C.L. region deduced from the data of the running period No.1 [4], the solid contour line delimits the 2- $\sigma$ C.L. region deduced from the data of the running period No. 2 [3], and the dashed contour line delimits the 2- $\sigma$ C.L. region, obtained by combining together the data of the two running periods. The solid open curve denotes the $90 \%$ C.L. upper bound, obtained in Ref. [5], where a pulse shape analysis of the events was used. This figure is reproduced from Fig. 6 of Ref. [3]. 
lation increases to $99.6 \%$ C.L. It is noticeable that the two sets of data have been taken under different operating conditions, since the experimental set-up was dismounted and reassembled between the two running periods.

In extracting the contour lines of Fig. 1 from the experimental data, the values of some astrophysical parameters (the root mean square velocity $v_{\text {rms }}$ of the WIMP Maxwellian velocity distribution in the halo, the WIMP escape velocity $v_{\text {esc }}$ in the halo, the velocity $v_{\odot}$ of the Sun around the galactic center), relevant for the event rates at the detector, had to be chosen. The values adopted in Fig. 1 refer to the median values of these parameters in their experimentally allowed ranges (reported, for instance, in Ref. [6]), namely, $v_{\text {rms }}$ $=270 \mathrm{~km} \mathrm{~s}^{-1}, v_{\mathrm{esc}}=650 \mathrm{~km} \mathrm{~s}^{-1}, v_{\odot}=232 \mathrm{~km} \mathrm{~s}^{-1}$.

In Refs. $[7,8]$ we derived the theoretical implications of the experimental data of Ref. [4], assuming that the indication of the possible annual modulation reported there was due to relic neutralinos. We selected the relevant supersymmetric configurations and discussed how these may be investigated by indirect searches for relic WIMPs and at accelerators.

In the present paper we apply a similar analysis to the new, much more significant set of data of Ref. [3] and we show that these data are fully compatible with an interpretation in terms of a relic neutralino as the major component of dark matter in the Universe. We pin down the regions of the supersymmetric parameter space relevant for this neutralino and derive the implications for search at accelerators.

A word of caution is in order here. As also remarked in Ref. [3], although the new DAMA data appear to bring more evidence for a possible annual modulation effect, first singled out in Ref. [4], this effect awaits further confirmation by additional experimental investigation in WIMP direct detection [9]. Actually, the DAMA-NaI Collaboration has already collected new data over the past year; moreover, the experiment still keeps running under good stability conditions [3] and is expected to provide increasingly significant statistics in the future. Furthermore, it is remarkable that, as subsequently discussed in the present paper, the supersymmetric configurations singled out by the annual modulation effect are also explorable at accelerators and in terms of indirect signals of relic neutralinos (i.e., in terms of antiprotons in space and of up-going muons at neutrino telescopes).

\section{SUPERSYMMETRIC MODEL}

In this paper we consider the neutralino as a WIMP candidate, able to induce annual modulation effects in direct particle dark matter searches. This supersymmetric particle is defined as the lowest-mass linear superposition of photino $(\tilde{\gamma})$, Z-ino $(\widetilde{Z})$ and the two Higgsino states $\left(\widetilde{H}_{\circ}{ }^{1}, \widetilde{H}_{\circ}{ }^{2}\right)[10]$ :

$$
\chi \equiv a_{1} \tilde{\gamma}+a_{2} \widetilde{Z}+a_{3} \widetilde{H}_{\circ}{ }^{1}+a_{4} \widetilde{H}_{\circ}{ }^{2} .
$$

We also define a parameter $P \equiv a_{1}^{2}+a_{2}^{2}$ in terms of which we classify neutralinos as gaugino-like when $P>0.9$, mixed when $0.1 \leqslant P \leqslant 0.9$, and Higgsino-like when $P<0.1$.

As a theoretical framework we use the minimal supersymmetric extension of the standard model (MSSM) [10], which conveniently describes the supersymmetric phenomenology at the electroweak scale, without too strong theoretical assumptions. This model has been extensively used by a number of authors for evaluations of the neutralino relic abundance and detection rates (a list of references may be found, for instance, in Ref. [7]).

The MSSM is based on the same gauge group as the standard model, contains the supersymmetric extension of its particle content and two Higgs doublets $H_{1}$ and $H_{2}$. As a consequence, the MSSM contains three neutral Higgs fields: two of them $(h, H)$ are scalar and one $(A)$ is pseudoscalar. At the tree level the Higgs sector is specified by two independent parameters: the mass of one of the physical Higgs fields, which we choose to be the mass $m_{A}$ of the neutral pseudoscalar boson, and the ratio of the two vacuum expectation values, defined as $\tan \beta \equiv\left\langle H_{2}\right\rangle /\left\langle H_{1}\right\rangle$. Once radiative corrections are introduced, the Higgs sector depends also on the squark masses through loop diagrams. The radiative corrections to the neutral and charged Higgs bosons, employed in the present paper, are taken from Refs. [11,12]. The other parameters of the model are defined in the superpotential, which contains all the Yukawa interactions and the Higgsmixing term $\mu H_{1} H_{2}$, and in the soft-breaking Lagrangian, which contains the trilinear and bilinear breaking parameters and the soft gaugino and scalar mass terms.

The MSSM contains a large number of free parameters. To cast it into a form adequate for phenomenology, it is necessary to introduce a number of restrictive assumptions at the electroweak scale. The usual conditions, which are also employed here, are the following: (i) all trilinear parameters are set to zero except those of the third family, which are unified to a common value $A$; (ii) all squarks and sleptons soft-mass parameters are taken as degenerate, $m_{\tilde{l}}=m_{\tilde{q} i}$ $\equiv m_{0}$, (iii) the gaugino masses are assumed to unify at $M_{\mathrm{GUT}}$, and this implies that the $\mathrm{U}(1)$ and $\mathrm{SU}(2)$ gaugino masses are related at the electroweak scale by $M_{1}$ $=(5 / 3) \tan ^{2} \theta_{W} M_{2}$.

After these conditions are applied, the supersymmetric parameter space consists of six independent parameters. We choose them to be $M_{2}, \mu, \tan \beta, m_{A}, m_{0}, A$ and vary these parameters in the following ranges: $10 \mathrm{GeV} \leqslant M_{2} \leqslant 500$ $\mathrm{GeV}, 10 \mathrm{GeV} \leqslant|\mu| \leqslant 500 \mathrm{GeV}, 75 \mathrm{GeV} \leqslant m_{A} \leqslant 1 \mathrm{TeV}$, $100 \mathrm{GeV} \leqslant m_{0} \leqslant 1 \mathrm{TeV},-3 \leqslant A \leqslant+3,1 \leqslant \tan \beta \leqslant 50$. We remark that the values taken here as upper limits of the ranges for the dimensional parameters $M_{2}, \mu, m_{0}, m_{A}$ are inspired by the upper bounds which may be derived for these quantities in supergravity (SUGRA) theories, when one requires that the electroweak symmetry breaking, radiatively induced by the soft supersymmetry breaking, does not occur with excessive fine tuning (see Ref. [13], and references quoted therein).

Our supersymmetric parameter space is further constrained by all the experimental limits obtained from accelerators on supersymmetric and Higgs boson searches. Thus, the latest data from the CERN $e^{+} e^{-}$collider LEP2 on Higgs boson, neutralino, chargino, and sfermion masses are used $[14,15]$. 
Moreover, the constraints due to the $b \rightarrow s+\gamma$ process (see, for instance, Refs. [16-21]) have to be taken into account. In our analysis, the inclusive decay rate $\operatorname{BR}(B$ $\left.\rightarrow X_{s} \gamma\right)$ is calculated with corrections up to the leading order. Next-to-leading order corrections [22-25] are included only when they can be applied in a consistent way, i.e., both to standard-model and to supersymmetry (SUSY) diagrams. This criterion limits the use of next-to-leading order corrections to peculiar regions of the supersymmetric parameter space, where the assumptions, under which the next-toleading order SUSY corrections have been obtained, apply [25]. We require that our theoretical evaluation for $\operatorname{BR}(B$ $\left.\rightarrow X_{s} \gamma\right)$ is within the range $1.96 \times 10^{-4} \leqslant \mathrm{BR}\left(B \rightarrow X_{s} \gamma\right)$ $\leqslant 4.32 \times 10^{-4}$. This range is obtained by combining the experimental data of Refs. $[26,27]$ at $95 \%$ C.L. and by adding a theoretical uncertainty of $25 \%$, whenever the still incomplete next-to-leading order SUSY corrections cannot be applied.

Since we are exploring here the neutralino as a stable dark matter candidate, we have to further constrain the parameter space by requiring that the neutralino is the lightest supersymmetric particle (LSP), i.e., we have to exclude regions where the gluino or squarks or sleptons are lighter than the neutralino. We also have to disregard those regions of the parameter space where the neutralino relic abundance exceeds the cosmological bound, derivable from measurements of the age of the Universe [28] and of the Hubble constant [29]. Conservatively, for this cosmological bound we take $\Omega_{\chi} h^{2} \leqslant 0.7$ [ $h$ is the usual Hubble parameter, defined in terms of the present-day value $H_{0}$ of the Hubble constant as $\left.h \equiv H_{0} /\left(100 \mathrm{~km} \mathrm{~s}^{-1} \mathrm{Mpc}^{-1}\right)\right]$. The neutralino relic abundance is calculated here as illustrated in Ref. [30]. Inclusion of coannihilation effects [31] in the calculation of $\Omega_{\chi} h^{2}$ are not necessary here, since the instances under which these effects might be sizeable are marginal in our supersymmetric parameter space.

\section{SELECTION OF SUPERSYMMETRIC CONFIGURATIONS BY THE ANNUAL MODULATION EFFECT}

We discuss now which region in the SUSY parameter space is selected by the new DAMA modulation data [3]. Let us start by converting the region delimited by the $2-\sigma$ C.L. dashed contour line in the plane $\xi \sigma_{\text {scalar }}^{\text {(nulleon }}-m_{\chi}$ of Fig. 1 into an enlarged one, which accounts for the uncertainty in the value of $\rho_{l}$. If a possible flattening of the dark matter halo [32] and a possibly sizeable baryonic contribution to the galactic dark matter [33] are taken into account, the following range for $\rho_{l}$ has conservatively to be taken: $0.1 \mathrm{GeV} \mathrm{cm} c^{-3} \leqslant \rho_{l} \leqslant 0.7 \mathrm{GeV} \mathrm{cm}{ }^{-3}$. One then obtains from the 2- $\sigma$ C.L. region of Fig. 1, where the total dark matter density is normalized to the value $\rho_{l}$ $=0.3 \mathrm{GeV} \mathrm{cm}{ }^{-3}$, the relevant 2- $\sigma$ C.L. region of Fig. 2 (hereafter denoted as region $R$ ).

Now we have to find which supersymmetric configurations, out of those in the parameter space outlined in Sec. II, are selected by the requirement that $\left(m_{\chi}, \xi \sigma_{\text {scalar }}^{(\text {nucleon })}\right) \in R$. To
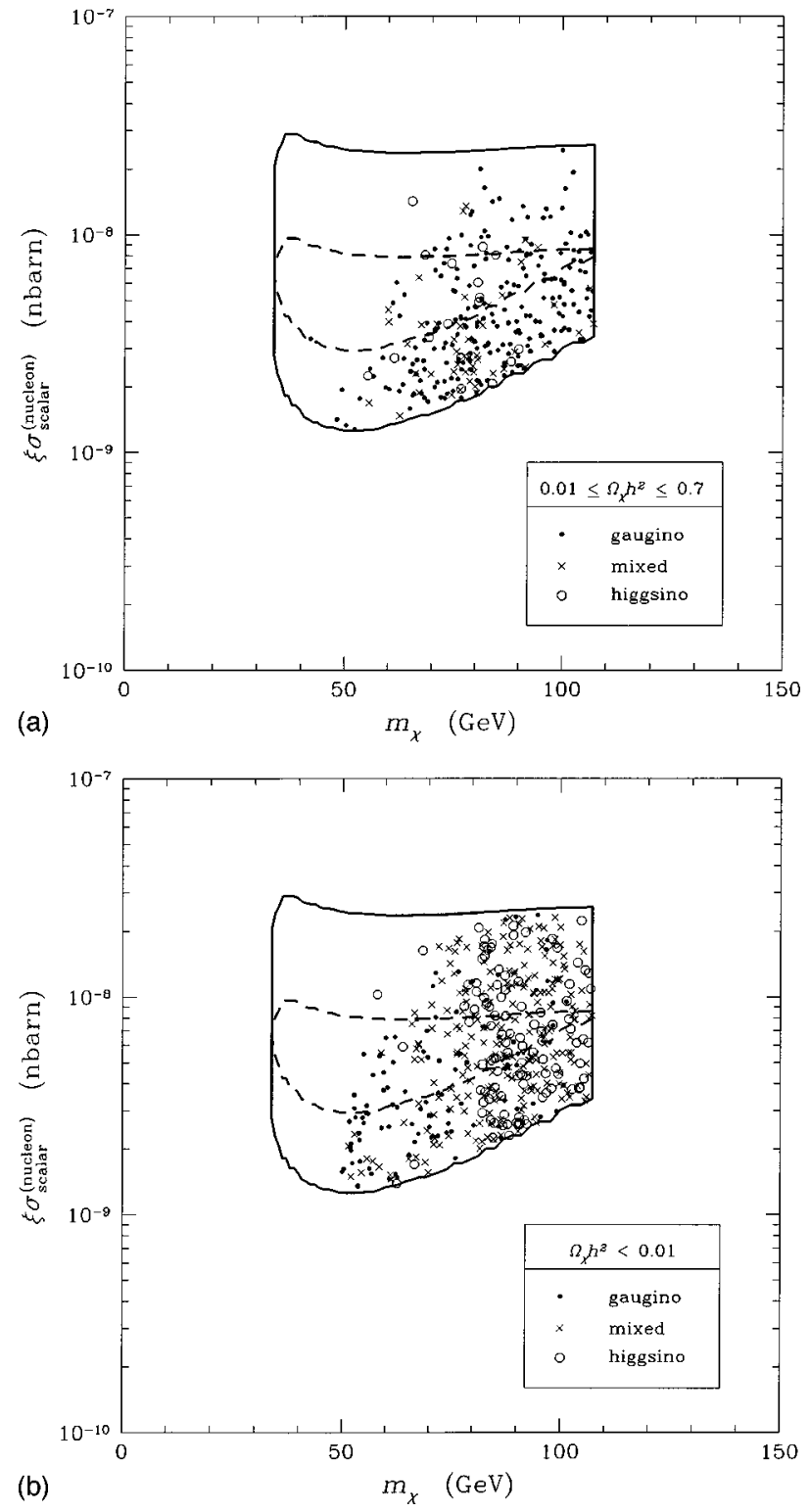

FIG. 2. Scatter plot of set $S$ in the plane $m_{\chi}-\xi \sigma_{\text {scalar }}^{\text {(nucleon) }}$. The dashed contour line delimits the 2- $\sigma$ C.L. region, obtained by the DAMA/NaI Collaboration, by combining together the data of the two running periods of the annual modulation experiment [3]. The solid contour line is obtained from the dashed line, which refers to the value $\rho_{l}=0.3 \mathrm{GeV} \mathrm{cm}{ }^{-3}$, by accounting for the uncertainty range of $\rho_{l}$, as explained in Sec. III (the region delimited by the solid line is denoted as region $R$ in the text). Displayed in this figure are only the representative points of the SUSY parameter space, defined in Sec. II, which fall inside the region $R$. Dots, crosses and circles denote neutralino compositions according to the classification given in Sec. II. (a) and (b) refer to configurations with 0.01 $\leqslant \Omega_{\chi} h^{2} \leqslant 0.7$ and with $\Omega_{\chi} h^{2}<0.01$, respectively.

this purpose we evaluate $m_{\chi}, \sigma_{\text {scalar }}^{\text {(nucleon }}$ and $\xi$ in the MSSM scheme previously defined. The neutralino mass is evaluated as usual by taking the lowest mass eigenstate of the neutralino mass matrix [10].

The neutralino-nucleon scalar cross section is calculated with the formula 


$$
\begin{aligned}
\sigma_{\text {scalar }}^{\text {(nucleon })}= & \frac{8 G_{F}^{2}}{\pi} M_{Z}^{2} m_{\text {red }}^{2}\left[\frac{F_{h} I_{h}}{m_{h}^{2}}+\frac{F_{H} I_{H}}{m_{H}^{2}}\right. \\
& \left.+\frac{M_{Z}}{2} \sum_{q}\langle N|\bar{q} q| N\rangle \sum_{i} P_{\tilde{q}_{i}}\left(A_{\tilde{q}_{i}}^{2}-B_{\tilde{q}_{i}}^{2}\right)\right]^{2},
\end{aligned}
$$

where the two first terms inside the brackets refer to the diagrams with $h$ and $H$ exchanges in the $t$ channel (the $A$-exchange diagram is strongly kinematically suppressed and then omitted here) [34] and the third term refers to the graphs with squark exchanges in the $s$ and $u$ channels [35]. The mass $m_{\text {red }}$ is the neutralino-nucleon reduced mass and

$$
\begin{gathered}
F_{h}=\left(-a_{1} \sin \theta_{W}+a_{2} \cos \theta_{W}\right)\left(a_{3} \sin \alpha+a_{4} \cos \alpha\right), \\
F_{H}=\left(-a_{1} \sin \theta_{W}+a_{2} \cos \theta_{W}\right)\left(a_{3} \cos \alpha-a_{4} \sin \alpha\right), \\
I_{h, H}=\sum_{q} k_{q}^{h, H} m_{q}\langle N|\bar{q} q| N\rangle .
\end{gathered}
$$

The angle $\alpha$ rotates $H_{1}^{(0)}$ and $H_{2}^{(0)}$ into $h$ and $H$, and the coefficients $k_{q}^{h, H}$ are given by $k_{u \text {-type }}^{h}=\cos \alpha / \sin \beta$ and $k_{u \text {-type }}^{H}=-\sin \alpha / \sin \beta$ for the up-type quarks, and by $k_{d \text {-type }}^{h}=-\sin \alpha / \cos \beta$ and $k_{d \text {-type }}^{H}=-\cos \alpha / \cos \beta$ for the down-type quarks. The matrix elements $\langle N|\bar{q} q| N\rangle$ are meant over the nucleonic state. By using the heavy quark expansion [36], one may rewrite the quantity $I_{h, H}$ as follows:

$$
I_{h, H}=k_{u \text {-type }}^{h, H} g_{u}+k_{d-\text { type }}^{h, H} g_{d},
$$

where

$$
\begin{aligned}
& g_{u}=\frac{4}{27}\left(m_{N}+\frac{19}{8} \sigma_{\pi N}-a \sigma_{\pi N}\right), \\
& g_{d}=\frac{2}{27}\left(m_{N}+\frac{23}{4} \sigma_{\pi N}+\frac{25}{2} a \sigma_{\pi N}\right) .
\end{aligned}
$$

Here $\sigma_{\pi N}$ is the so-called pion-nucleon sigma term, $\sigma_{\pi N}$ $=\frac{1}{2}\left(m_{u}+m_{d}\right)\langle N|\bar{u} u+\bar{d} d| N\rangle$, and the parameter $a$ is related to the strange-quark content of the nucleon $y$ by

$$
a=y \frac{m_{s}}{m_{u}+m_{d}}, \quad y=2 \frac{\langle N|\bar{s} s| N\rangle}{\langle N|\bar{u} u+\bar{d} d| N\rangle} .
$$

For these parameters we use the following values: $\sigma_{\pi N}$ $=45 \mathrm{GeV}$ [37], $y=0.33 \pm 0.09$ [38], and $2 m_{s} /\left(m_{u}+m_{d}\right)$ $=29$ [39]; thus, using the central value of $y$, we obtain $g_{u}$ $=123 \mathrm{GeV}$ and $g_{d}=288 \mathrm{GeV}$.

In the squark-exchange terms of Eq. (2) $\Sigma_{i}$ denotes a sum over the mass eigenstates, $P_{\tilde{q}_{i}}$ stands for the squark propagators

$$
P_{\tilde{q}_{i}}=\frac{1}{2}\left(\frac{1}{m_{\tilde{q}_{i}}^{2}-\left(m_{\chi}-m_{q}\right)^{2}}+\frac{1}{m_{\tilde{q}_{i}}^{2}-\left(m_{\chi}+m_{q}\right)^{2}}\right),
$$

and the $A_{\tilde{q}_{i}}$ and $B \tilde{q}_{i}$ coefficients are given by

$$
\begin{aligned}
A_{\tilde{q}_{1}} & =\cos \theta_{q}\left(X_{q}+Z_{q}\right)+\sin \theta_{q}\left(Y_{q}+Z_{q}\right), \\
B_{\tilde{q}_{1}} & =\cos \theta_{q}\left(X_{q}-Z_{q}\right)+\sin \theta_{q}\left(Z_{q}-Y_{q}\right), \\
X_{q} & =-\left(\cos \theta_{W} T_{3 q} a_{2}+\sin \theta_{W} \frac{Y_{q L}}{2} a_{1}\right), \\
Y_{q} & =\sin \theta_{W} \frac{Y_{q R}}{2} a_{1}, \\
Z_{u \text {-type }} & =-\frac{m_{u \text {-type }} a_{4}}{2 \sin \beta M_{Z}}, \\
Z_{d \text {-type }} & =-\frac{m_{d \text {-type }} a_{3}}{2 \cos \beta M_{Z}},
\end{aligned}
$$

where $T_{3 q}, Y_{q L}, Y_{q R}$ refer to the isospin and to the hypercharge quantum numbers of $\tilde{q}_{L, R}$, respectively. The couplings $A_{\tilde{q}_{2}}$ and $B_{\tilde{q}_{2}}$ may be obtained with the substitution $\sin \theta_{q} \rightarrow \cos \theta_{q}$ and $\cos \theta_{q} \rightarrow-\sin \theta_{q}$.

In our numerical applications the squark propagators in Eq. (7) have been regularized by inserting appropriate widths in the denominators. In general, it turns out that the Higgsexchange amplitudes are largely dominant over the squarkexchange ones, the latter competing with the former ones almost exclusively when an enhancement in their size is originated by a mass fine-tuning in the squark-propagator denominators.

As for the values to be assigned to the quantity $\xi$ $=\rho_{\chi} / \rho_{l}$ we adopt the standard rescaling recipe [40]. For each point of the parameter space, we take into account the relevant value of the cosmological neutralino relic density. When $\Omega_{\chi} h^{2}$ is larger than a minimal value $\left(\Omega h^{2}\right)_{\min }$, compatible with observational data and with large-scale structure calculations, we simply put $\xi=1$. When $\Omega_{\chi} h^{2}$ turns out to be less than $\left(\Omega h^{2}\right)_{\min }$, and then the neutralino may only provide a fractional contribution $\Omega_{\chi} h^{2} /\left(\Omega h^{2}\right)_{\text {min }}$ to $\Omega h^{2}$, we take $\xi=\Omega_{\chi} h^{2} /\left(\Omega h^{2}\right)_{\min }$. The value to be assigned to $\left(\Omega h^{2}\right)_{\min }$ is somewhat arbitrary, in the range 0.01 $\lesssim\left(\Omega h^{2}\right)_{\min } \lesssim 0.3$. We use here the value $\left(\Omega h^{2}\right)_{\min }=0.01$, which is conservatively derived from the estimate $\Omega_{\text {galactic }}$ $\sim 0.03$.

Using the previous formulas we find that a large portion of the modulation region $R$ is indeed covered by supersymmetric configurations, compatible with all present physical constraints. This set of SUSY states, which will hereafter be denoted as set $S$, is displayed in Fig. 2 with different symbols, depending on the neutralino composition. In Fig. 2(a) we notice that a quite sizeable portion of region $R$ is populated by supersymmetric configurations with neutralino relic abundance inside the cosmologically interesting range 0.01 $\lesssim \Omega_{\chi} h^{2} \lesssim 0.7$. Thus we obtain the first main result of our analysis, i.e., the annual modulation region, singled out by the DAMA-NaI experiment, is largely compatible with a relic neutralino as the major component of dark matter. This is certainly the most remarkable possibility. However, we also keep under consideration neutralino configurations with 


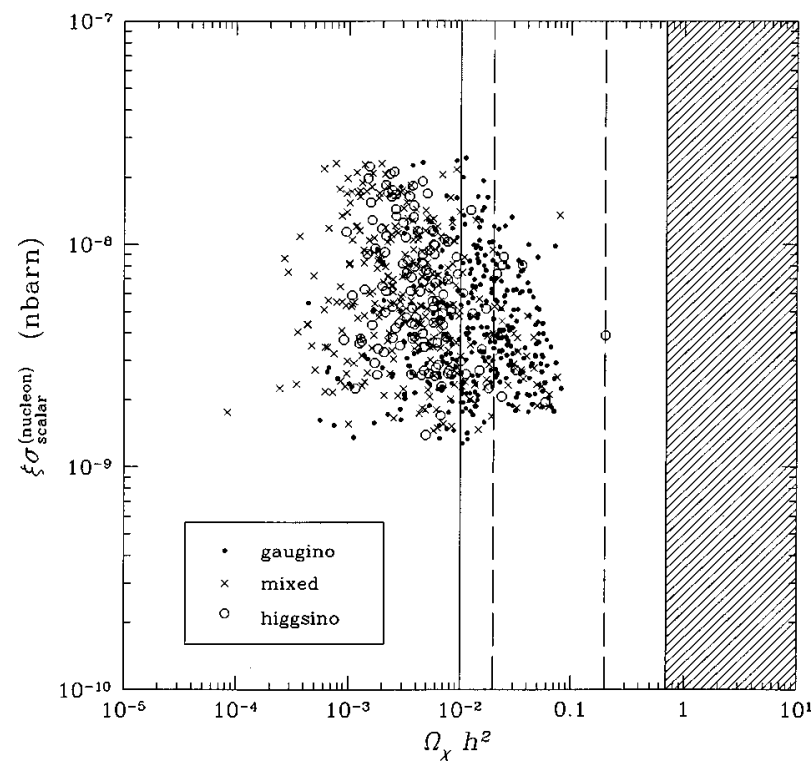

FIG. 3. Scatter plot of set $S$ in the plane $\Omega_{\chi} h^{2}-\xi \sigma_{\text {scalar }}^{\text {(nuclen) }}$. Dots, crosses, and circles denote neutralino compositions according to the classification given in Sec. II. The two vertical solid lines delimit the $\Omega_{\chi} h^{2}$-range of cosmological interest. The two dashed lines delimit the most appealing interval for $\Omega_{\chi} h^{2}$, as suggested by the most recent observational data.

a small contribution to $\Omega_{\chi} h^{2}$ [see Fig. 2(b)], since also the detection of relic particles with these features would provide in itself a very noticeable information.

The neutralino relic abundance $\Omega_{\chi} h^{2}$ is plotted versus the quantity $\xi \sigma_{\text {scalar }}^{\text {(nucleon) }}$ in terms of the neutralino composition in Fig. 3. Here we remark some anticorrelation between the two plotted quantities. This feature is expected on general grounds, as discussed, for instance, in Ref. [41]. In fact, it is due to the combination of two properties: (i) the direct detection rate is proportional to $\sigma_{\text {scalar }}^{\text {(nucleon) }}$ and $\Omega_{\chi} h^{2} \propto \sigma_{\text {ann }}^{-1}$, where $\sigma_{\text {ann }}$ is the neutralino-neutralino annihilation cross section, (ii) usually $\sigma_{\text {ann }}$ and $\sigma_{\text {scalar }}^{\text {(nuclen) }}$, as functions of the supersymmetric model parameters, are either both increasing or both decreasing. Therefore, neutralinos with lower values for the relic abundance have higher couplings with matter (this feature is attenuated, when rescaling in $\rho_{\chi}$ is operative; this occurs here for $\Omega_{\chi} h^{2}<0.01$ ).

In view of the discussed anticorrelation between $\sigma_{\text {scalar }}^{\text {(nucleon }}$ and $\Omega_{\chi} h^{2}$, it is remarkable that the relatively large neutralino-matter cross sections, implied by the DAMA modulation effect, agree with a relic neutralino making up a major contribution to dark matter, i.e., with a neutralino whose relic abundance falls into the cosmologically interesting range $0.01 \leq \Omega_{\chi} h^{2} \leq 0.7$. Most of the neutralino configurations falling in this range of $\Omega_{\chi} h^{2}$ turn out to be gauginolike.

We further notice that recent observations and analyses [42] point to values of $\Omega_{\text {matter }}$ somewhat smaller than those considered in the past: $0.1 \leq \Omega_{\text {matter }} \leqslant 0.4$. If we combine this range with the one for $h: 0.55 \leqq h \leqq 0.80$ [29] and require that a cold dark matter candidate (such as the neutralino) supplies $\sim(80-90) \%$ of $\Omega_{\text {matter }}$, we obtain $0.02 \lesssim \Omega_{\mathrm{CDM}} h^{2}$ $\lessgtr 0.2$. This turns out to be the most appealing interval for relic neutralinos. It is remarkable that this range for $\Omega_{\chi} h^{2}$ is densely populated by configurations of set $S$ (see Fig. 3 ).

\section{FURTHER PROPERTIES OF THE CONFIGURATIONS SINGLED OUT BY THE ANNUAL MODULATION EFFECT}

Let us proceed now to an analysis of other main properties of the configurations of set $S$, related to a possible investigation of these supersymmetric states at accelerators.

As is already clear from Fig. 2, the set $S$ contains neutralino compositions of various nature, from Higgsino-like to gaugino-like ones. This property is further displayed in Fig. 4 , where we show the location of the configurations of set $S$ in the plane $\mu-M_{2}$, for two representative values of $\tan \beta$.

The properties of our set $S$ relevant to searches of neutral Higgs bosons at accelerators are displayed in Fig. 5. (a) shows a scatter plot of set $S$ in term of $m_{h}$ and $\tan \beta$, (b) provides essentially the same information, but in terms of $m_{h}$ and the quantity $\sin ^{2}(\alpha-\beta)$, which is the relevant coupling for the channels of possible neutral Higgs boson production at LEP. In the plot of section (a) it is apparent a correlation between $\tan \beta$ and $m_{h}$. This is due to the fact that the rather large values of the neutralino-nucleon scalar cross section, $\sigma_{\text {scalar }}^{\text {(nuclen) }} \sim\left(10^{-9}-10^{-8}\right) \mathrm{nb}$, as required by the annual modulation data, impose that either the couplings are large (then large $\tan \beta$ ) and/or the process goes through the exchange of a light particle. Thus, Higgs-exchange dominance and $\sigma_{\text {scalar }}^{\text {(nucleon }} \sim\left(10^{-9}-10^{-8}\right) \mathrm{nb}$ require a very light $h$ at small $\tan \beta$, and even put a lower bound on $\tan \beta: \tan \beta$ 22.5 . At larger values of $\tan \beta$, the mass $m_{h}$ is less constrained, also because, at large $\tan \beta$, the squark-exchange diagrams may occasionally compete with the Higgsexchange ones in keeping $\xi \sigma_{\text {scalar }}^{\text {(nucleon }}$ at a sizeable value. From Fig. 5(a) we notice that a good deal of SUSY configurations are explorable at LEP2, while others will require experimental investigation at a high luminosity Fermilab Tevatron, which should be capable to explore Higgs boson masses up to $m_{h} \sim 130 \mathrm{GeV}[43,44]$.

In Fig. 6 the configurations of set $S$ are shown in the plane $m_{\tilde{t}_{1}}-\tan \beta$ ( $\tilde{t}_{1}$ denotes the lightest top squark). This scatter plot reveals an interesting correlation: at small $\tan \beta$ only light $\tilde{t}_{1}$ 's are allowed. In the Appendix it is shown that this feature occurs as a joint effect due to the $b \rightarrow s+\gamma$ constraint and to the annual modulation data [45].

From the previous results, it then turns out that annual modulation data and $b \rightarrow s+\gamma$ constraint complement each other in providing stringent bounds on both $m_{h}$ and $m_{\tilde{t}_{1}}$, at small $\tan \beta$. For instance, for $\tan \beta \leqq 5$ one has $m_{h}$ $\lesssim 105 \mathrm{GeV}$ and $m_{\tilde{t}_{1}} \leqslant 350 \mathrm{GeV}$.

Finally, in Fig. 7 we display the scatter plot of set $S$ in the plane $m_{\chi}$-tan $\beta$. Since the reach of LEP2 extends only up to the dashed vertical line, at $m_{\chi} \simeq 50 \mathrm{GeV}$, the exploration of the whole interesting region will require Tevatron upgrades or LHC. Under favorable hypothesis, TeV33 could provide exploration up to the vertical solid line.

Apart from exploration at accelerators, configurations of 

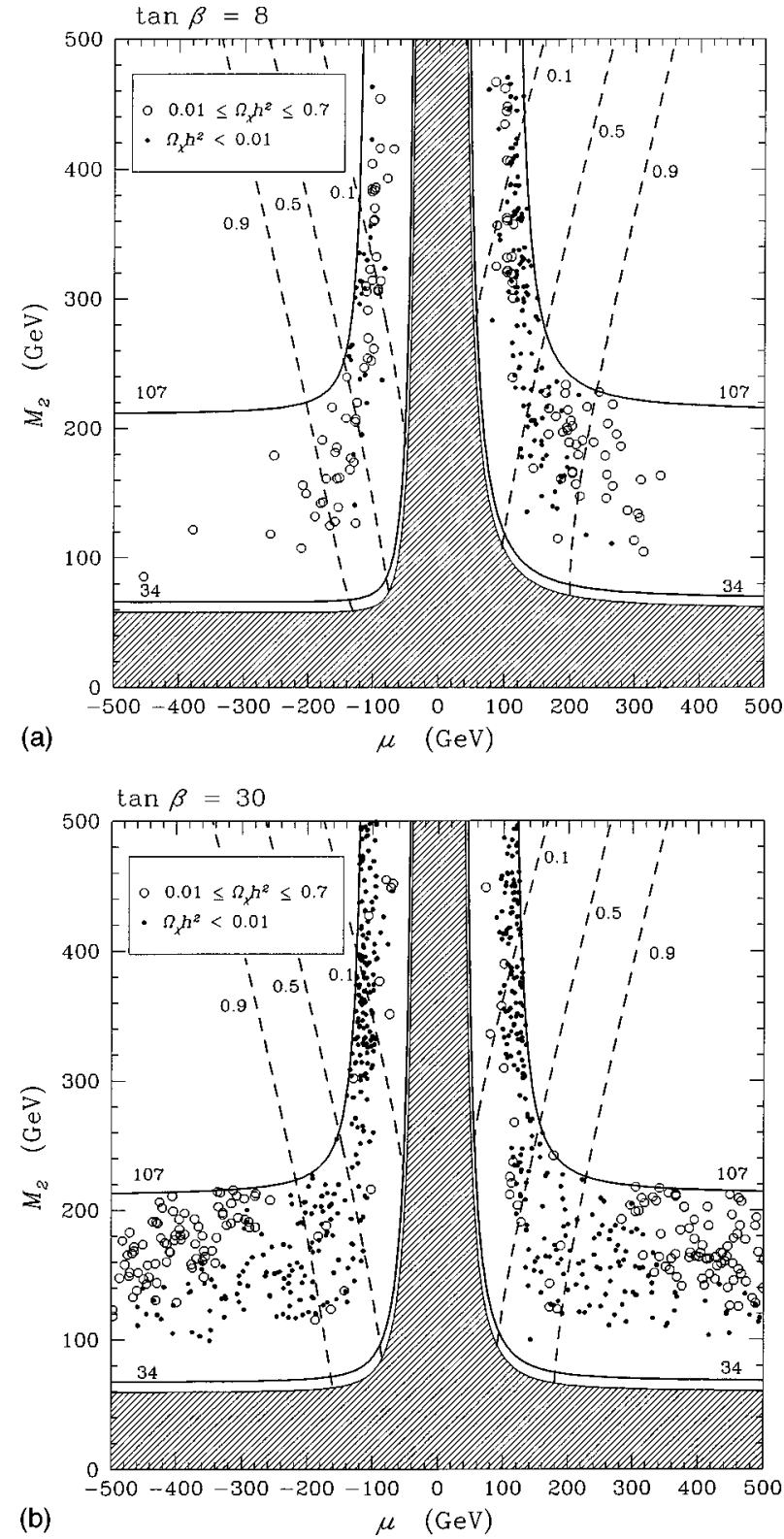

FIG. 4. Scatter plot of set $S$ in the plane $\mu-M_{2}$. (a) and (b) refer to two representative values of $\tan \beta: \tan \beta=8$ and $\tan \beta=30$, respectively. The solid curves denote the isomass curves which delimit the annual modulation region $R$, i.e., the isomass curves for $m_{\chi}=34 \mathrm{GeV}$ and $m_{\chi}=107 \mathrm{GeV}$. The dashed curves denote the neutralino composition, and correspond to $P=0.1,0.5,0.9$. The hatched region is excluded by LEP at $\sqrt{s}=183 \mathrm{GeV}$.

set $S$ may be investigated by means of indirect measurements of relic neutralinos, such as cosmic-ray antiprotons [46] and neutrino fluxes from Earth and Sun (Ref. [41], and references quoted therein). On the basis of a preliminary analysis, we found that configurations of set $S$ provide quite significant signals in both instances. In the case of antiprotons, a large fraction of configurations of set $S$ provide $\bar{p}$ fluxes at the level of the measurements by the balloon-borne BESS experiment [47]. These configurations will be further investigated with the data collected during the Shuttle flight by the AMS experiment [48]. A similar situation occurs for the neu-
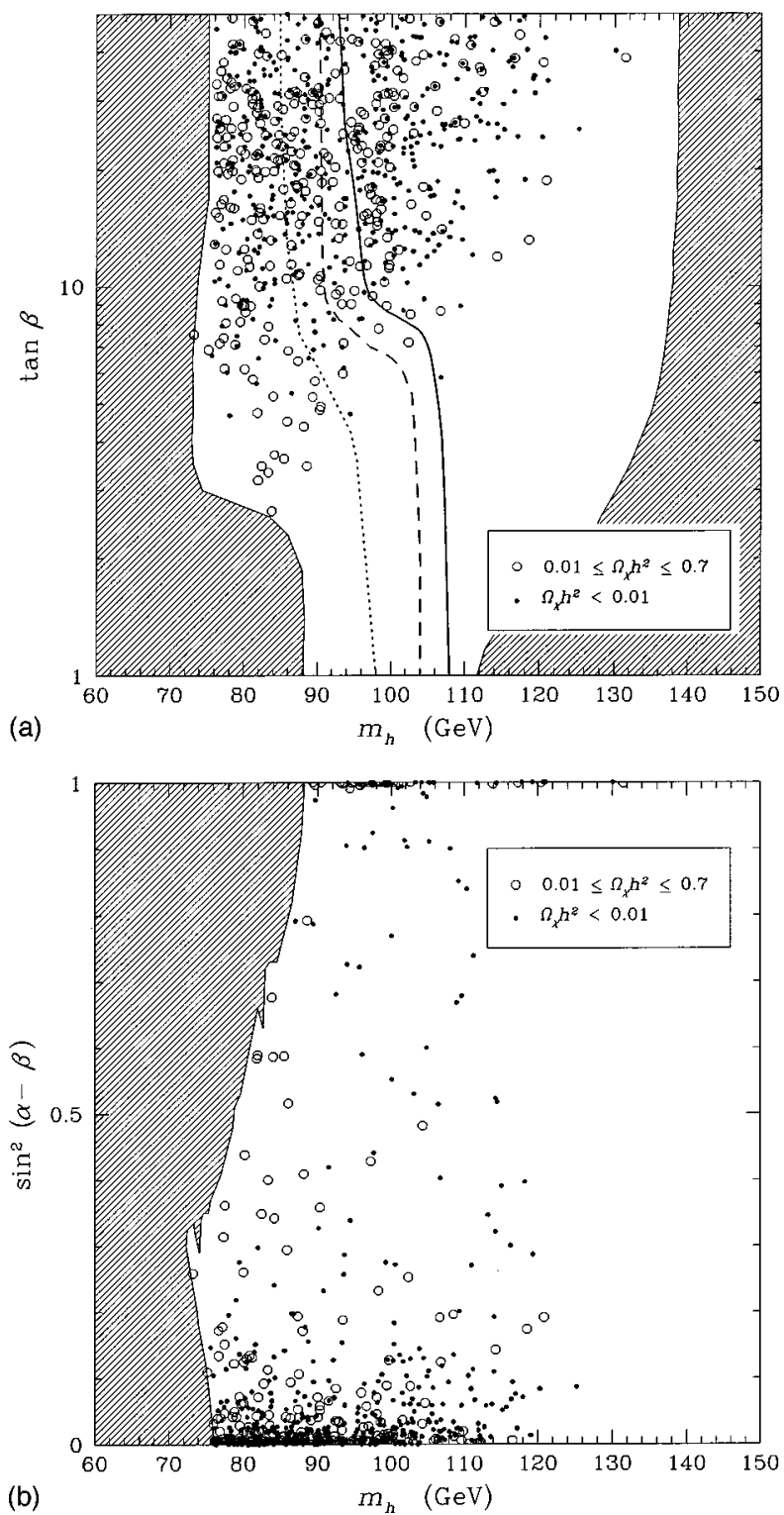

FIG. 5. (a) Scatter plot for set $S$ in the plane $m_{h}-\tan \beta$. The hatched region on the right is excluded by theory. The hatched region on the left is excluded by present LEP data at $\sqrt{s}$ $=183 \mathrm{GeV}$. The dotted and the dashed curves denote the reach of LEP2 at energies $\sqrt{s}=192 \mathrm{GeV}$ and $\sqrt{s}=200 \mathrm{GeV}$, respectively. The solid line represents the $95 \%$ C.L. bound reachable at LEP2, in case of non discovery of a neutral Higgs boson. (b) Scatter plot for set $S$ in the plane $m_{h}-\sin ^{2}(\alpha-\beta)$. The hatched region on the left is excluded by LEP data at $\sqrt{s}=183 \mathrm{GeV}$.

trino fluxes induced by configurations of set $S$, which turn out to be within the reach of MACRO [49] and Baksan [50] neutrino telescopes. Details of our analysis on the indirect detection searches are presented in Ref. [51].

We end this section by some more general theoretical considerations. We have discussed here the physical implications of the annual modulation data in the framework of a MSSM at the electroweak scale, since this scheme provides the simplest and least-constrained model for discussing SUSY phenomenology. However, we have also performed an analysis of the modulation data in the framework of su- 


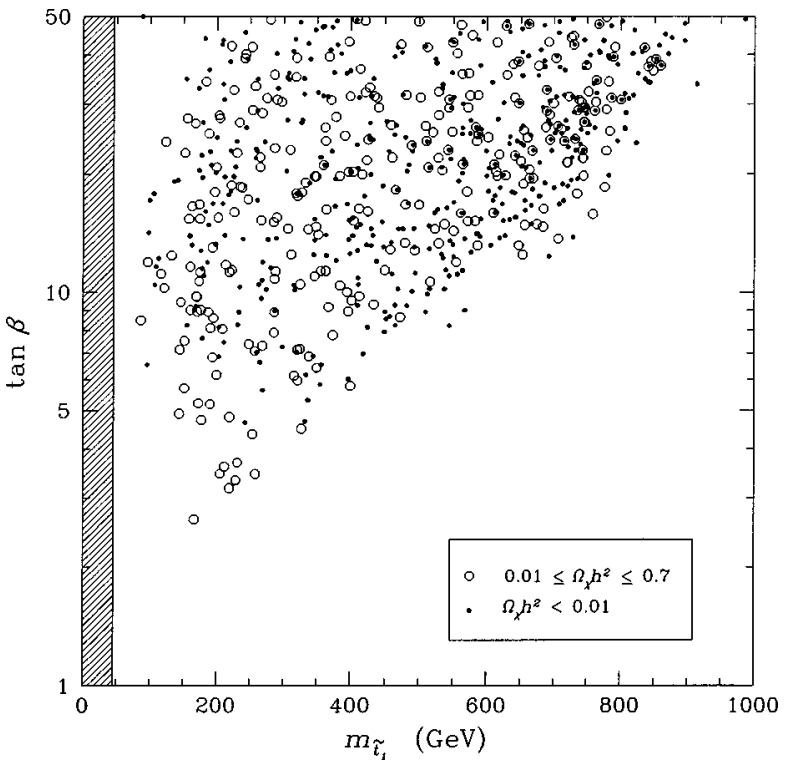

FIG. 6. Scatter plot for set $S$ in the plane $m_{\tilde{t} 1}-\tan \beta$. The hatched region is excluded by LEP data (without any restriction on other masses).

pergravity (SUGRA) theories. The results of this study are presented in Ref. [52]. We simply report here that we have ascertained that a fraction of configurations of set $S$ are indeed compatible with SUGRA schemes, even more so when the unification conditions, which are usually imposed at grand unified theory (GUT) scale, are somewhat relaxed, for instance by allowing deviations from a strict unification assumption in the Higgs boson masses at the GUT scale [13]. It is remarkable that these configurations fall into the region of SUSY parameter space where electroweak symmetry breaking occurs without excessive fine tuning between com-

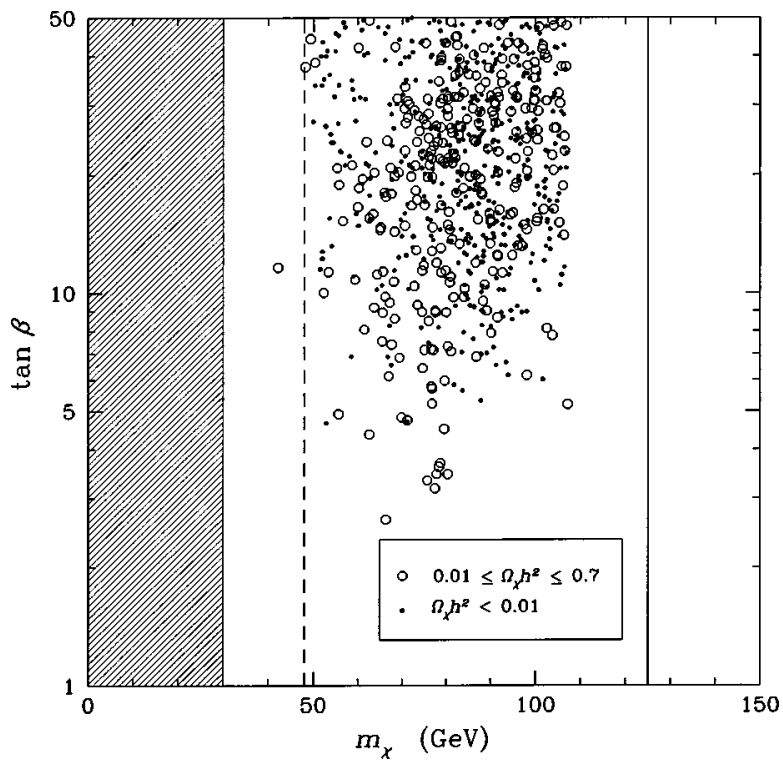

FIG. 7. Scatter plot for set $S$ in the plane $m_{\chi}$-tan $\beta$. The hatched region on the left is excluded by present LEP data. The dashed and the solid vertical lines denote the reach of LEP2 and TeV33, respectively.

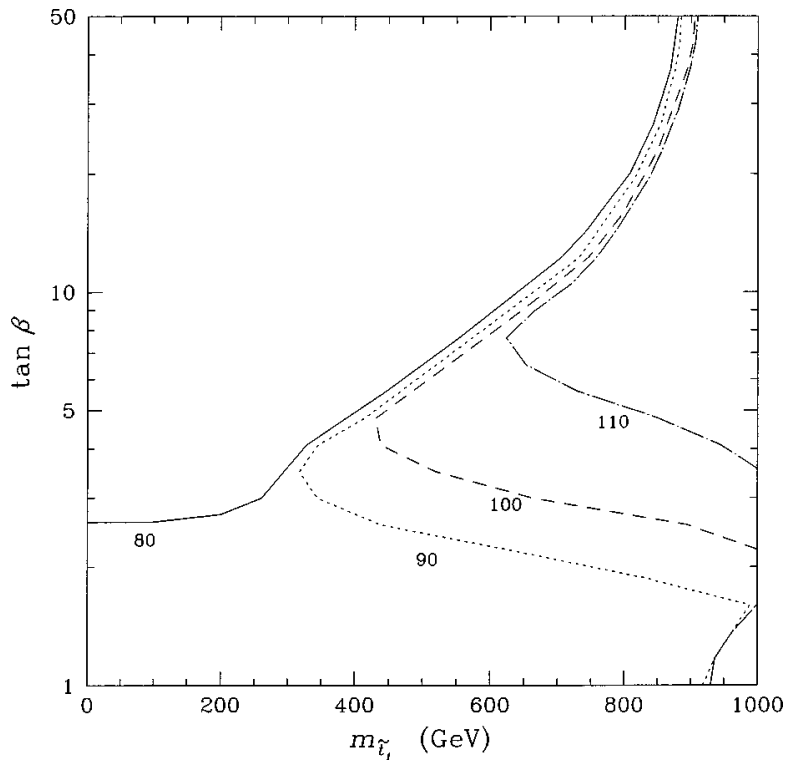

FIG. 8. Regions of the parameter space defined in Sec. II, which satisfy all accelerator constraints (including $b \rightarrow s+\gamma$ ) and the further requirement that $m_{h}$ is below some arbitrarily fixed value $m_{h}^{*}$. The various lines denote the following representative values of $m_{h}^{*}: m_{h}^{*}=80,90,100,110 \mathrm{GeV}$. The allowed regions are given by the domains on the left of the various curves for each value of $m_{h}^{*}$.

peting terms. A simple case of this feature occurs for the neutralino mass, whose range for the annual modulation configurations is well within the no-fine-tuning upper bound $m_{\chi} \leq \mathrm{O}(100 \mathrm{GeV})[13]$.

\section{CONCLUSIONS}

The new data of the DAMA/NaI experiment [3], which support a possible annual modulation effect in the counting rates for relic WIMPs, previously reported by the same Collaboration [4], have been analyzed here in terms of relic neutralinos. We have proved that the annual modulation data are largely compatible with a relic neutralino making up the major part of dark matter in the Universe.

We have also investigated the possibility of exploring the supersymmetric states, selected by the annual modulation data, at accelerators. We have demonstrated that an analysis of the main features of these SUSY configurations is within the reach of present or planned experimental setups. In particular, we have found the following results

(a) The sizeable neutralino-nucleon elastic cross sections, implied by the annual modulation data, entail a rather stringent upper bound for $m_{h}$ in terms of $\tan \beta$. In particular, this property implies that no SUSY configuration would be allowed for $\tan \beta \lesssim 2.5$. A large portion of the region covered by the scatter plot in the plane $m_{h}$-tan $\beta$ is explorable at LEP2, the remaining one will be at $\mathrm{TeV} 33$.

(b) The annual modulation data and the $b \rightarrow s+\gamma$ constraint complement each other in providing a correlation between $\tan \beta$ and the mass of the lightest top squark.

As remarked in the introduction, a solid confirmation of the annual modulation effect as singled out by the DAMA- 
$\mathrm{NaI}$ Collaboration will require further accumulation of an increasingly significant statistics with very stable setups over a few years. However, it is worth noticing that the detection of this effect, if confirmed by further experimental evidence, would turn out to be a major breakthrough in establishing the existence of particle dark matter in the Universe. It is very rewarding that the features of this dark matter particle are widely compatible with those expected for the neutralino, both in MSSM and in SUGRA schemes, and that several of its properties can be explored in the near future at accelerators and by indirect searches for relic neutralinos.

\section{ACKNOWLEDGMENTS}

We wish to thank Professor R. Bernabei and Dr. P. Belli for very interesting discussions about experimental aspects of the DAMA/NaI experiment and about the analysis of their data. We also thank Dr. P. Gambino for informative discussions on the next-to-leading order corrections to $b \rightarrow s+\gamma$.

\section{APPENDIX}

Here we discuss the origin of the correlation between $m_{t_{1}}$ and $\tan \beta$ which is apparent in the plot of Fig. 6 at small $\tan \beta$. Let us start by considering how the $b \rightarrow s+\gamma$ constraint [16-21] correlates the three parameters $\tan \beta, m_{h}$, and $m_{\tilde{t}_{1}}$. Thus, leaving momentarily aside the annual modulation data, let us consider in the plane $m_{\tilde{t}_{1}}-\tan \beta$ the regions of our parameter space which satisfy all accelerator constraints (including $b \rightarrow s+\gamma$ ) and the further requirement that $m_{h}$ is below some arbitrarily fixed value $m_{h}^{*}$. In Fig. 8 these regions are represented by the domains on the left of the various lines, which are denoted by the following values of $m_{h}^{*}: m_{h}^{*}=80,90,100,110 \mathrm{GeV}$. It is possible to show that

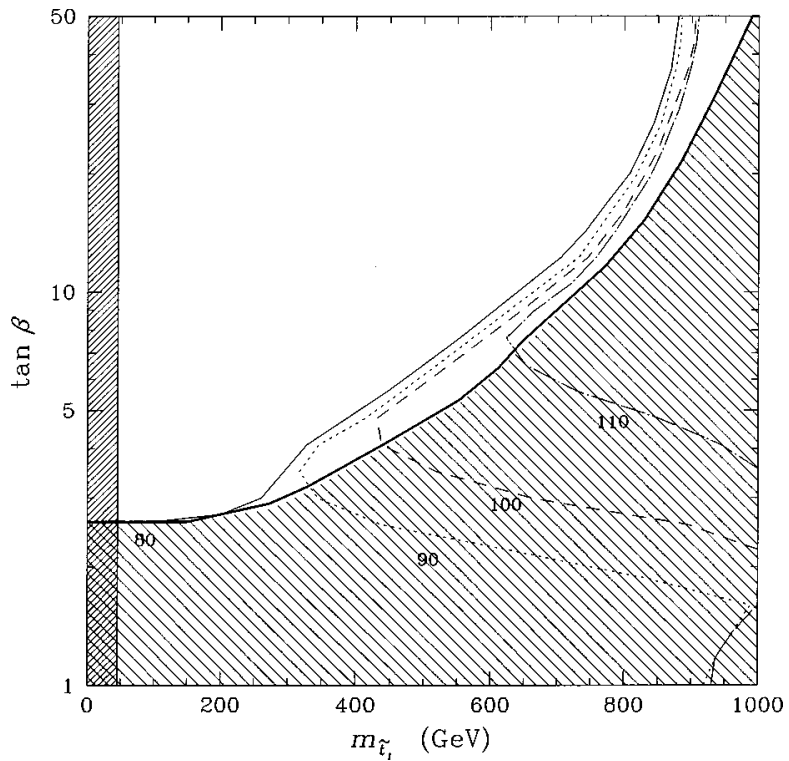

FIG. 9. Allowed region in the plane $m_{\tilde{t}_{1}}-\tan \beta$ when the plot of Fig. 8 is combined with the one of Fig. 5(a). The hatched region on the left is excluded by LEP data (without any restriction on other masses).

the $b \rightarrow s+\gamma$ constraint is instrumental in establishing the peculiar shape of the various contour lines at fixed $m_{h}$.

If we now combine the plot of Fig. 8 with the one of Fig. 5(a) we obtain the situation displayed in Fig. 9, the allowed region being the one on the left of the various curves, depending on the values of $m_{h}^{*}$. From this figure we see how the $\tan \beta-m_{\tilde{t}_{1}}$ correlation, occurring in Fig. 6, is due to the joint effect of $b \rightarrow s+\gamma$ and annual modulation data.
[1] A.K. Drukier, K. Freese, and D.N. Spergel, Phys. Rev. D 33, 3495 (1986).

[2] K. Freese, J. Frieman, and A. Gould, Phys. Rev. D 37, 3388 (1988).

[3] R. Bernabei et al., Phys. Lett. B (to be published), Roma II University Report No. ROMA2F/98/27; Riv. Nuovo Cimento (to be published), ROMA2F/98/34, 1998.

[4] R. Bernabei et al., Phys. Lett. B 424, 195 (1998).

[5] R. Bernabei et al., Phys. Lett. B 389, 757 (1996).

[6] A. Bottino, F. Donato, G. Mignola, S. Scopel, P. Belli, and A. Incicchitti, Phys. Lett. B 402, 113 (1997).

[7] A. Bottino, F. Donato, N. Fornengo, and S. Scopel, Phys. Lett. B 423, 109 (1998).

[8] A. Bottino, F. Donato, N. Fornengo, and S. Scopel, Torino University Report No. DFTT 61/97, hep-ph/9710295, 1997.

[9] For updated reviews on direct search experiments see, for instance, D.O. Caldwell and A. Morales, in TAUP 97, Proceedings of the International Workshop on Topics in Astroparticle and Underground Physics, Gran Sasso, Italy, 1997, edited by A. Bottino et al. [Nucl. Phys. B (Proc. Suppl.) 70, 43 (1999)]; ibid., p. 54.
[10] H.P. Nilles, Phys. Rep. 110, 1 (1984); H.E. Haber and G.L. Kane, ibid. 117, 75 (1985); R. Barbieri, Riv. Nuovo Cimento 11, 1 (1988).

[11] M. Carena, M. Quirós, and C.E.M. Wagner, Nucl. Phys. B461, 407 (1996).

[12] H.E. Haber, Z. Phys. C 75, 539 (1997).

[13] V. Berezinsky, A. Bottino, J. Ellis, N. Fornengo, G. Mignola, and S. Scopel, Astropart. Phys. 5, 1 (1996).

[14] M. Felcini, in Workshop "'From Planck Scale to Electroweak Scale,' Kazimierz, 1998 (unpublished); A. Lipniacka, ibid.; ALEPH Collaboration, R. Barate et al., Phys. Lett. B 433, 176 (1998); L3 Susy Group, L3 Note 2238 (unpublished).

[15] K. Desch and P. Rebecchi, talks at International Conference on High Energy Physics, Vancouver, 1998 (unpublished).

[16] S. Bertolini, F. Borzumati, A. Masiero, and G. Ridolfi, Nucl. Phys. B353, 591 (1991).

[17] R. Barbieri and G.F. Giudice, Phys. Lett. B 309, 86 (1993).

[18] R. Garisto and J.N. Ng, Phys. Lett. B 315, 372 (1993).

[19] F.M. Borzumati, M. Drees, and M.M. Nojiri, Phys. Rev. D 51, 341 (1995). 
[20] J. Wu, R. Arnowitt, and P. Nath, Phys. Rev. D 51, 1371 (1995).

[21] V. Barger, M.S. Berger, P. Ohmann, and R.J.N. Phillips, Phys. Rev. D 51, 2438 (1995).

[22] K. Chetyrkin, M. Misiak, and M. Münz, Phys. Lett. B 400, 206 (1997).

[23] M. Ciuchini, G. Degrassi, P. Gambino, and G.F. Giudice, Nucl. Phys. B527, 21 (1998).

[24] A. Czarnecki and W.J. Marciano, Phys. Rev. Lett. 81, 277 (1998).

[25] M. Ciuchini, G. Degrassi, P. Gambino, and G.F. Giudice, Nucl. Phys. B534, 3 (1998).

[26] CLEO Collaboration, S. Glenn, Report No. CLEO CONF 9817, 1998; International Conference on High Energy Physics, Vancouver, 1998, paper 1011 (unpublished).

[27] ALEPH Collaboration, R. Barate et al., Phys. Lett. 129B, 169 (1998).

[28] B. Chaboyer, P. Demarque, P. Kernan, and L.M. Krauss, Astrophys. J. 494, 96 (1998).

[29] A. Sandage et al., Astrophys. J. Lett. 460, L15 (1996); W.L. Freedman, talk at the 18th Texas Symposium, Chicago, 1996, astro-ph/9706072, and references quoted therein.

[30] A. Bottino, V. de Alfaro, N. Fornengo, G. Mignola, and M. Pignone, Astropart. Phys. 2, 67 (1994).

[31] P. Binétruy, G. Girardi, and P. Salati, Nucl. Phys. B237, 285 (1984); K. Griest and D. Seckel, Phys. Rev. D 43, 3191 (1991); S. Mizuta and M. Yamaguchi, Phys. Lett. B 298, 120 (1993); J. Edsjö and P. Gondolo, Phys. Rev. D 56, 1879 (1997).

[32] E. Gates, G. Gyuk, and M.S. Turner, Phys. Rev. Lett. 74, 3724 (1995); Astrophys. J. Lett. 449, L123 (1995); Phys. Rev. D 53, 4138 (1996).

[33] E. Gates, G. Gyuk, and M.S. Turner, talk presented at 18th Texas Symposium on Relativistic Astrophysics, Chicago, 1996, astro-ph/9704253.

[34] R. Barbieri, M. Frigeni, and G.F. Giudice, Nucl. Phys. B313, 725 (1989).

[35] K. Griest, Phys. Rev. D 38, 2357 (1988); Phys. Rev. Lett. 61, 666 (1988).

[36] M.A. Shifman, A.I. Vainshstein, and V.I. Zacharov, Phys. Lett. 78B, 443 (1978); JETP Lett. 22, 55 (1975).

[37] J. Gasser, H. Leutwyler, and M.E. Sainio, Phys. Lett. B 253, 252 (1991).

[38] S.J. Dong and K.F. Liu, in Lattice '94, Proceedings of the International Symposium, Bielefeld, Germany, edited by F.
Karsch et al. [Nucl. Phys. B (Proc. Suppl.) 42, 322 (1995)].

[39] J. Bijnens, J. Prades, and E. de Rafael, Phys. Lett. B 348, 226 (1995).

[40] T.K. Gaisser, G. Steigman, and S. Tilav, Phys. Rev. D 34, 2206 (1986).

[41] V. Berezinsky, A. Bottino, J. Ellis, N. Fornengo, G. Mignola, and S. Scopel, Astropart. Phys. 5, 333 (1996).

[42] W.L. Freedman, R. Kirshner, and C. Lineweaver, talks given at the International Conference of Cosmology and Particle Physics, Geneva, 1998, http://wwwth.cern.ch/capp98/ programme.html; M. White, Astrophys. J. 506, 495 (1998); N.A. Bahcall and X. Fan, astro-ph/9804082; C. Lineweaver, astro-ph/9805326.

[43] Report of the TeV-2000 Study Group, edited by D. Amidei and R. Brock, Fermilab Report No. FERMILAB-PUB96/082, 1996.

[44] H. Baer, B.W. Harris, and X. Tata, Phys. Rev. D 59, 015003 (1998).

[45] The relevance of the $b \rightarrow s+\gamma$ constraint for WIMP direct detection rates (without considering annual modulation effect) has been discussed in the past in a number of papers with some conflicting conclusions. See, for example, F.M. Borzumati, M. Drees, and M.M. Nojiri, Phys. Rev. D 51, 341 (1995); P. Nath and R. Arnowitt, Phys. Rev. Lett. 74, 4592 (1995); V. Berezinsky, A. Bottino, J. Ellis, N. Fornengo, G. Mignola, and S. Scopel, Astropart. Phys. 5, 1 (1996); L. Bergström and P. Gondolo, ibid. 5, 263 (1996); V.A. Bednyakov, S.G. Kovalenko, H.V. Klapdor-Kleigrothaus, and Y. Ramachers, Z. Phys. A 357, 339 (1997).

[46] A. Bottino, F. Donato, N. Fornengo, and P. Salati, Phys. Rev. D 58, 123503 (1998).

[47] BESS Collaboration, H. Matsunaga et al., in Proceedings of the 25th International Conference of Cosmic Rays, Durban, 1997, edited by H. S. Potgieter, B. C. Raubenheimer, and D. J. van der Walt (World Scientific, Singapore, 1998).

[48] X Ahlen et al., Nucl. Instrum. Methods Phys. Res. A 350, 351 (1994).

[49] MACRO Collaboration, M. Ambrosio et al., Report No. MACRO/PUB1/98; T. Montaruli et al., in TAUP 97 [9], p. 367.

[50] M.M. Boliev et al., TAUP 97 [9], p. 371.

[51] A. Bottino, F. Donato, N. Fornengo, and S. Scopel, Torino University Report No. DFTT 49/98, 1998 [Astropart. Phys. (to be published)].

[52] A. Bottino, F. Donato, N. Fornengo, and S. Scopel, following paper, Phys. Rev. D 59, 095004 (1999). 